\title{
Provision of Critical Maternity Care during the Coronavirus Disease 2019 (COVID-19) Pandemic in the Islamic Republic of Iran
}

\author{
Nasrin Changizi, MD'; Alireza Raeisi, MD²; Hamed Barekati, MD³; Abbas Habibollahi, MD; Haniye Sadat Sajadi, PhD; Nezhat Emami- \\ Afshar, MSc ${ }^{6}$; Laleh Radpooyan, MSc ${ }^{6}$

\begin{abstract}
'Maternal, Fetal and Neonatal Research Center, Tehran University of Medical Sciences, Tehran, Iran \& Maternal Health Department, Population, Family and School Health Office, Deputy of Health, Ministry of Health and Medical Education, Tehran, Iran

${ }^{2}$ Shiraz University of Medical Sciences, Shiraz, Iran \& Deputy of Health, Ministry of Health and Medical Education, Tehran, Iran ${ }^{3}$ Population, Family and School Health Office, Deputy of Health, Ministry of Health and Medical Education, Tehran, Iran ${ }^{4}$ Neonatal Health Department, Population, Family and School Health Office, Deputy of Health, Ministry of Health and Medical Education, Tehran, Iran

${ }^{5}$ Knowledge Utilization Research Center, University Research and Development Center, Tehran University of Medical Sciences, Tehran, Iran ${ }^{6}$ Maternal Health Department, Population, Family and School Health Office, Deputy of Health, Ministry of Health and Medical Education, Tehran, Iran
\end{abstract}

\begin{abstract}
Reducing maternal mortality is one of the Sustainable Development Goals. Although there is no vigorous evidence that pregnant women are in the high-risk groups in response to coronavirus disease 2019 (COVID-19), it is crucial to respond to the pandemic through providing required action plans for confirmed or suspected pregnant women cases while maintaining routine functions. Iran's response and preparedness measures to COVID-19 aimed to meet the essential needs required to protect pregnant women and their families. Establishing a national maternal health network, relying on mechanisms for timely reporting, monitoring, and following-up, preparing guidelines and protocols required for COVID-19 management in pregnant women though a multidisciplinary team working approach, and embedding the precautions of reducing transmission in maternity care were the main measures taken to cope with COVID-19 in pregnancy. Iran's experience in providing maternity care during the COVID-19 can guide other countries affected by COVID-19. However, it should be adapted to local health-care facilities, as well as in response to any further updates on COVID-19.

Keywords: COVID-19, Disease outbreaks, Epidemics, Maternal health services, Mortality, Pregnancy

Cite this article as: Changizi N, Raeisi A, Barekati H, Habibollahi A, Sajadi HS, Emami-Afshar N, et al. Provision of critical maternity care during the coronavirus disease 2019 (COVID-19) pandemic in the Islamic Republic of Iran. Arch Iran Med. 2020;23(8):557-560. doi: 10.34172/aim.2020.59.
\end{abstract}

Received: April 11, 2020, Accepted: June 2, 2020, ePublished: August 1, 2020

\section{Introduction}

Coronavirus disease 2019 (COVID-19) is an emerging communicable disease with a rapid increase in many countries since its first identification in December 2019. ${ }^{1}$ The available information shows that some people, including older adults and people with severe chronic medical conditions, are at higher risk of becoming very sick from this illness. ${ }^{2,3}$ There is no vigorous evidence that pregnant women are more susceptible to infection, or at increased risk for severe disease, morbidity, mortality with COVID-19, or adverse pregnancy or infant outcome compared with others. However, due to their immunologic and physiologic changes, it is recommended to consider pregnant women as one of the vulnerable groups. ${ }^{1}$

The first case of COVID-19 in the Islamic Republic of (IR) Iran was officially reported on February 19, $2020 .{ }^{4}$ Following that, the country's efforts have begun to tackle the ongoing COVID-19 epidemic. ${ }^{5}$ Most efforts have been led by the Ministry of Health and Medical Education (MOHME), whose primary mission is to provide high-quality health care for all Iranian. Different departments of MOHME have developed their programs for taking action against COVID-19. Since IR Iran is trying hard to meet the targets of maternal health defined by Sustainable Development Goals (SDGs), taking immediate measures to provide maternal care during the pandemic of COVID-19 disease was a top priority. So, with early warning signs in the country, the maternal health programs focused mainly on COVID-19 management in pregnancy.

In this manuscript, we describe the IR Iran's response to 
provide maternal care during the COVID-19 pandemic that aimed to provide care for those infected with or suspected of COVID-19, and to ensure that no mother was left behind because of the fear of the pandemic. It may help to share knowledge on COVID-19 response and preparedness measures being implemented in countries affected by COVID-19.

\section{Iran's Response to Provide Maternal Care during COVID-19 Pandemic}

Given the tough situation experienced to protect pregnant women with $\mathrm{H} 1 \mathrm{~N} 1$, and the news of COVID-19 outbreak in China, the National Maternal Health Committee (NMHC) started preliminary efforts to develop an action plan to respond to any respiratory infection problems in pregnant women. Following the announcement of the first case of COVID-19 in IR Iran, these efforts were scaled up and focused on the appropriate response to COVID-19. We explain these efforts regarding the four steps of preventing infections, saving lives, and minimizing impact. ${ }^{6}$

Step 1: Preparedness

- Three committees have been established to prepare everyone for the response to infectious diseases. The first committee was formed in mid-December with more than 60 maternal health technical officers of Curative Deputies of Medical Universities (MUs). The second one was established in late December with more than 60 maternal health technical officers of Health Deputies of MUs. The last one was created in mid-February with representatives of 10 mega-region MUs which supervise all 60 MUs. Representatives were of different fields, including intensivists, obstetricians, gynecologists, perinatologists, and midwives. Some members of the NMHC participate in this committee, as well. While the committee had its meeting to discuss COVID-19, the first case of COVID-19 in IR Iran was launched by the national authorities. Following that, the extraordinary meeting of the committee was held on February 23 to draft and finalize the maternal health protocols during the COVID-19 pandemic, based on the previous meetings. Only representatives of the three MUs located at Tehran, as capital city, participated in this meeting, due to restrictions on nonessential travel. Required protocols and guidelines were prepared and sent to the authorized body in MOHME. They were launched to all MUs a few days later.

- The National Maternal Health Networking (NMHN) was established. Different virtual groups were formed in NMHN, including

- The national group with representatives of 10 mega-region MUs, including maternal health technical officers in Curative and Health
Deputies and head of obstetricians in Educational Deputies: This group is in charge of leading and coordinating all MUc and having direct connection with them;

- The multidisciplinary maternal health groups at national and local levels with experts selected from different fields of expertise (obstetrician/ fetomaternal fellowship, neonatologist, pulmonologist, intensivists/anesthesiologist, radiologist, infectious disease specialist, cardiologist, clinical pharmacologist, forensic medicine specialist). While the National group provides all MUs with technical advice and support to address the challenges of managing pregnant women, the local groups in the MUs are responsible for patient management in their own area.

○ Ten regional groups in each mega-region MUs with representatives of related MUs in each region. They are responsible for supervision and coordination.

- The local groups in each MUs with representatives of the university. These groups have been asked to be in close connection with related region group and to solve problems related to maternal health in their circle or otherwise to send their questions to regional or national groups.

- Several protocols, guidelines, and flowcharts were developed to ensure providing safe care during the COVID-19 pandemic. They include case finding in pregnant women, maternal care during labor and postpartum, inpatient services for suspect/ confirmed COVID-19 pregnant women in hospitals, distance working for pregnant health providers, suspect/confirmed COVID-19 triage in pregnancy (revised almost every two weeks), how to approach intrapartum/antenatal care suspect/confirmed pregnant case in hospitals, and cardiac dysfunction in COVID-19 pregnant women. Modifications were made to the national integrated health software to adapt it to new protocols;

- An official request for personal protective equipment (PPE) was sent to international agencies;

- A project was designed and implemented to gather the required data for decision-making purpose;

- The National Radiology Association was asked to reassess the CT findings of suspect/confirmed COVID-19 in some cases of pregnant women;

- The National Maternal Mortality Surveillance system was temporarily revised to omit outdoor verbal autopsy.

- The Echocardiography Association in collaboration with NMHC was asked to develop indications for cardiologic consultation and echocardiographic request. 
Step 2: Detect, Protect and Treat

- All MUs were asked to report all cases of pregnant women who are admitted to hospitals. It was emphasized to pay more attention to those who are hospitalized in intensive care units (ICUs). A reporting form was prepared through the MOHME Intranet Platform that helps MUs to report suspected/infected cases immediately. By April 1, 2020, 182 pregnant women with confirmed and 150 with suspected COVID-19 have been hospitalized, of whom 30 and 9 pregnant women have been transferred to ICUs, and 18 and 6 pregnant women have been discharged, respectively.

- The NMHC virtually follows all pregnant women with confirmed or suspected COVID-19 hospitalized in ICUs. The NMHC, in collaboration with the multidisciplinary national maternal health group, provides all technical help and advice for MUs involved.

- A follow-up form was designed for those who were discharged or planned to be cared for at home up to 42 days postpartum for confirmed cases. By April 1, 2020, 260 confirmed COVID-19 pregnant women were discharged from hospitals, and 51 confirmed outpatient pregnant cases were followed up for at least 14 days after becoming asymptomatic.

Step 3: Reduce Transmission

- All MUs have been asked since February 24 to add a question to the clinical assessment of confirmed COVID-19 cases about having a pregnant woman in their household or neighborhood.

- All health care providers, including midwives, obstetricians and pediatrician/neonatologist were requested to use PPE when visiting confirmed/ suspected pregnant women with COVID-19, with particular attention during normal vaginal delivery as well as any surgical intervention for mothers.

- The number of antenatal care visits was temporarily decreased to minimize contact and exposure for pregnant women.

- All related scientific associations were asked for adherence to all protocols of COVID-19 management in pregnancy.

- Home visits were restricted.

- Technical officers providing extra-services for highrisk pregnant women in public sectors were asked for meticulous follow up of those high-risk pregnant women infected with COVID-19.

- If there is a need for organizing meetings to discuss problems and exchange experience, the sessions will be held online in most cases.

Step 4: Innovate and Learn

- The virtual NMHN, as mentioned above, was established to facilitate experience and information exchange.

- A virtual research team with medical students from different disciplines (e.g., medicine, pharmacy, obstetrics \& gynecology, reproductive health) was formed to conduct research projects and prepare educational materials based on the latest evidence.

- A multidisciplinary approach was employed to develop new protocols and guidelines.

- The public are informed about the COVID-19 in pregnancy through multimedia (e.g., video clips).

- Several educational modalities were prepared and distributed; short health messages are sent to pregnant mothers registered in the health network.

In conclusion, a resilient health system should respond effectively to the COVID-19 pandemic through withstanding health shocks while maintaining routine functions. ${ }^{7}$ Iran's response and preparedness measures to COVID-19 in pregnancy are an experience of how to fight shocks while the routine care is being provided. The most important measures were those taken to establish governance and coordination of multidisciplinary mechanisms to complement response protocols, identify context-relevant essential maternal health services, establish patient flow at all levels of service delivery arrangement, identify mechanisms to maintain availability of essential medications, equipment and supplies, and monitoring and reporting systems. In an era where evidence regarding the risks, outcomes and management of COVID-19 infection is scarce, Iran's experience in providing maternity care during the COVID-19 pandemic can guide other countries affected by COVID-19. However, these measures should be adapted to local health-care facilities, as well as in response to any further updates on COVID-19.

\section{Authors' Contribution}

The present report was the NC, AR, HB, AH and HSS idea. They have developed the framework of the report. NC and HSS wrote the first draft. NEA and LR worked in subsequent drafts. All authors confirmed the last version before submission. The authors read and approved the final manuscript.

\section{Conflict of Interest Disclosures \\ None.}

\section{Ethical Statement}

Not applicable.

Funding

The work was done with no financial support.

\section{References}

1. Luo Y, Yin K. Management of pregnant women infected with COVID-19. Lancet Infect Dis. 2020;20(5):513-514. doi: 10.1016/S1473-3099(20)30191-2.

2. World Health Organization. Report of the WHO-China Joint Mission on Coronavirus Disease 2019 (COVID-19). Geneva: WHO; 2020. 
3. World Health Organization. Coronavirus disease 2019 (COVID-19): situation report, 51. 2020. Available from: https://apps.who.int/iris/handle/10665/331475.

4. Arab-Mazar Z, Sah R, Rabaan AA, Dhama K, RodriguezMoralesAJ. Mapping the incidence of the COVID-19 hotspot in Iran - Implications for Travellers. Travel Med Infect Dis. 2020;34:101630. doi: 10.1016/j.tmaid.2020.101630.

5. Raeisi A, Tabrizi JS, Gouya MM. IR of Iran National Mobilization against COVID-19 epidemic. Arch Iran Med.
2020;23(4):216-9. doi: 10.34172/aim.2020.01.

6. World Health Organization. Available from: https://www. who.int/dg/speeches/detail/who-director-general-s-opening-remarks-at-the-media-briefing-on-covid-19---11-march-2020.

7. World Health Organization. Operational guidance for maintaining essential health services during an outbreak. Available from: https://www.who.int/publications-detail/ covid-19-operational-guidance-for-maintaining-essentialhealth-services-during-an-outbreak. 